\title{
THE LICK OBSERVATORY TWO MICRON CAMERA
}

\author{
K. Gilmore, D. Rank and P. Temi \\ Lick Observatory/UCO UCSC
}

\begin{abstract}
Lick Observatory has recently developed a near infrared camera for astronomical imaging. The new camera has been built around a Rockwell NICMOSII 256 x $256 \mathrm{HgCdTe}$ focal plane array. The dewar and optics were manufactured by Infrared Laboratories in Tucson, Arizona while the electronics and data system were designed and fabricated at Lick Observatory.
\end{abstract}

The new instrument was designed to image faint galaxies and other astronomical objects as well as embedded infrared sources in star formation regions. The camera has cold focal plane scales of $0.2,0.4$ and 0.6 arcseconds per pixel when used at the telescope. Standard $\mathrm{H}, \mathrm{J}$ and $\mathrm{K}$ as well as narrow band special purpose filters are contained in two independent motorized filter wheels.

The camera performance with different plate scales and filters as well as the mechanical, optical and electrical lay-out will be shown. M 42 will be used for characterization of the camera's performance. 


\section{DISCUSSION}

D'ODORICO: What is the best image quality that you have achieved at the telescope?

GILMORE: This past summer with URC2 at the Lick 3-m telescope, the best image taken was about 0.7.

FINGER: Is the lens close to the entrance window a cold lens?

GILMORE: Yes, the first element of the three element collimator is in contact with the radiation shield.

FINGER: Do you see a reset anomaly? How long does it take for the integration ramp to settle and become linear after reset?

GILMORE: In CDS mode we do not see an anomaly. In multi read mode it is seen. Several $\mu \mathrm{s}$ are required after reset for the ramp to become linear.

FLORENTIN-NIELSON: You have ball bearings inside the liquid nitrogen device. Do you have outgassing from any lubricant in these bearings?

GILMORE: The bearings do not use any lubricant. They are spring loaded into position onto a surface of like material. 